\title{
LA DIFFÉRENCE PHÉNOMÉNOLOGIQUE SELON BARBaras ET MARION (Projet, méthode et ligne de tension)
}

\author{
Eric Pommier ${ }^{1}$
}

Résumé: Cet article se propose de proposer les conditions d'une confrontation entre la phénoménologie de la donation de Jean-Luc Marion et l'ontologie de la vie de Renaud Barbaras. Cela suppose d'établir un certain plan de convergence à propos du projet d'une description de l'apparaître pur, de la méthode et de la conception "événementiale" du phénomène et du sujet afin de faire valoir une divergence quant à la question de la naissance transcendantale du sujet.

Mots-clé: Apparaître. Différence. Evénement. Naissance. Réduction.

\section{INTRODUCTION}

Un sourire n'est jamais qu'un sourire et l'on aurait peut-être tort d'y voir autre chose que cela que ce qui, dans ce phénomène, se donne, à savoir le plissement de lèvres exprimant un contentement réel ou feint. Pourtant, nous sentons bien aussi qu'en présence d'une certaine manière de sourire, à l'écoute d'une mélodie particulière, en lisant telle page de littérature ou de philosophie ou bien en recevant un singulier présent, quelque chose se passe qui semble nous conduire en-deçà ou au-delà du simple phénomène, de son contenu, comme si quelque chose en lui l'excédait, qu'il signifiait davantage que son être-là, que son être advenu.

Non pas qu'il nous conduise à un ordre d'être supérieur, supraempirique et pour tout dire métaphysique, comme si l'épreuve de certains objets permettait l'ascension jusqu’à un autre ordre de réalité qui transcende

\footnotetext{
${ }^{1}$ Professeur associé et directeur de la recherche de l'Institut de Philosophie de l'Université Pontificale Catholique du Chili, Santiago, Chile. (D) https://orcid.org/0000-0001-6675-4321 Email: epommier@uc.cl
}

http://doi.org/10.1590/0101-3173.2020.v43n3.08.p111

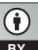

This is an open-access article distributed under the terms of the Creative Commons Attribution License. 
absolument le donné sensible pour nous faire découvrir, au plan de la connaissance rationnelle, ce qu'ils signifient véritablement.

Si dans ce sourire, il y a décidément plus que ce sourire, il n'y a en effet pas autre chose que ce sourire. Cela qui transcende le sourire ne va paradoxalement guère au-delà de lui, et la promesse de bonheur qu'il peut ouvrir n'offre rien d'autre que cette promesse. Ce surcroît phénoménal, qui n'est pas de l'ordre du supra-phénoménal, n’est-il donc rien? Faut-il donc renoncer à décrire ce jene-sais-quoi qui ne semble pouvoir être connu, comme l'on connaît un objet, ou compris comme l'on comprend un étant du monde? Peut-on, en toute bonne conscience, passer à côté de ce qui semble se passer au motif de ce qu'il est inconnaissable, incompréhensible et que le registre métaphysique ne lui est pas adéquat? Comme l'écrit Vladimir Jankélévitch (1986, p. 143):

Aussi le mystère n'est-il pas quelque chose d'existant dont nous ignorons encore le nom, comme c'est le cas du secret, mais un rien à jamais impensable dont nous sentons seulement l'absence ou la présence. Aussi peut-on sans risque feindre de méconnaître, ce qu'il n'est nullement indispensable de savoir (car, en fait, il n'y a rien à "savoir") et que chacun de nos actes, chacune de nos pensées pourtant présupposent.

Néanmoins, il ne faut sans doute pas trop vite renoncer à penser ce je-ne-sais-quoi. Puisque nous sommes sensibles à cette différence au sein du champ phénoménal, et qu'elle fait sens pour notre vie, il n'y a pas lieu de considérer que la description de cette différence soit a priori impossible, sous prétexte qu'elle ne semble pas renvoyer à une différence d'ordre métaphysique, épistémologique (celui du sujet et de l'objet) ou ontologique (celui de l'être et de l'étant.) Le mystère du sourire n'est mystère qu'au regard des modes de rationalité connus de nous: les principes de contradiction, de raison suffisante, d'objectivation. Puisque nous faisons l'expérience de cette étrange différence, il s'agit de penser selon cette expérience, sans lui imposer des concepts qui ne sont pas faits pour elle, et en lui en substituant d'autres à sa mesure.

Il se pourrait alors que le secret de ces phénomènes, qui semblent en excès au regard d'eux-mêmes, provient de ce qu'en eux, ou par eux, se donne le mouvement en vertu duquel ils nous apparaissent et s'adressent à nous. Traces tremblantes d'un passage en train de se produire, si ces phénomènes peuvent parfois nous donner une impression de rêve, comme s'ils n'existaient pas vraiment ou pas suffisamment, c'est peut-être que nous sommes prisonniers d'une tendance rigide à les appréhender ontiquement alors que c'est au plan 
purement phénoménal et dynamique qu'il faudrait s'y rapporter, afin de découvrir à travers eux cet excès dont ils sont porteurs et par lequel ils se manifestent pour nous.

Or, il nous semble que c'est cette prétention commune à rendre compte de la différence phénoménologique comme telle, sans la rabattre sur les différences métaphysiques, "épistémologiques" et ontologiques, qui permet de rapprocher les philosophies se faisant de Jean-Luc Marion et de Renaud Barbaras, et c'est ce que nous nous proposons de montrer dans cet article en adoptant naturellement une méthode phénoménologique qui se heurte aux limites de la phénoménologie traditionnelle. Dans les deux cas, il s'agirait de rendre compte du mouvement grâce auquel la manifestation comme telle, ou en soi, nous advient et par laquelle nous advenons à nous-mêmes en montrant le monde et en nous découvrant à nous-mêmes à cette occasion. Si une telle hypothèse n'est pas d'entrée de jeu erronée, il convient pour l'expliciter d'opérer au moins en quatre temps. Dans un premier moment, après avoir confirmé en quel sens c'est la question de l'accès à l'apparaitre comme tel à travers ce qui apparait qui permet de réunir les deux perspectives (section 1), il importera d'examiner la méthode qui permet à chacun des deux philosophes de se frayer une voie vers cet apparaître (section 2), avant de procéder à sa description comme cela qui advient, ce qui impliquera une redéfinition de la subjectivité (section 3). Notre réflexion de caractère analogique ne pourra cependant être menée à bien sans mettre également en relief au moins une ligne de tension qui oppose les démarches des deux phénoménologues français à propos de la naissance du sujet. Ce sera un résultat de cette recherche de restituer les conditions de ce débat entre eux à l'égard du problème de la naissance du sujet (section 4).

\section{LE PROBLÈME DE L'APPARAÎTRE COMME TEL. LA DIFFÉRENCE PHÉNOMÉNOLOGIQUE}

Indiquons, en premier lieu, en quel sens c'est bien la manière d'aborder la question de la manifestation en-deçà de la différence "épistémologique" du sujet et de l'objet et ontologique de l'être et de l'étant, qui autorise un rapprochement entre les démarches de Jean-Luc Marion et Renaud Barbaras.

Comme Jean-Luc Marion le montre dans le premier chapitre d'Etant Donné (MARION, 2013), sur la base de ce qui a été établi dans Réduction et Donation, la question de l'apparaître reste dominée chez Husserl, tout au long de son œuvre et en dépit de ses évolutions, par "le principe des principes" 
de l'intuition (HUSSERL, 1950, p. 78). Tout ce qui apparait se donne "en chair et en os", si et seulement s'il se donne dans une intuition qui remplit l'intention de signification. Or, si ce principe a l'avantage de révoquer en doute toute justification métaphysique de la phénoménalité, puisqu'il ne prétend pas chercher la source de cette dernière dans un principe qui lui serait extérieur et transcendant, les Idées platoniciennes par exemple, il a cependant le caractère d'une décision a priori qui pré-décide de la forme de ce qui doit apparaître au lieu de laisser le phénomène se donner comme tel, pour ce qu'il est. Au lieu de rester fidèle à l'exigence de décrire le comment de l'apparaître, Husserl réduit la scène phénoménale au seul champ de l'objet, réifie l'apparaître, réduit le donné à cela seul qui se donne dans une intuition d'objet. S'il est donc approprié de ne pas chercher l'essence de la chose qui se donne à la conscience en dehors de cette donation, à savoir dans une réalité supra-phénoménale, comme si l'essence de la chose devait être séparée de l'attestation de son existence, il n'en reste pas moins que l'a priori universel de corrélation husserlien, qui relie la chose à ses modes de donation subjectifs, ne permet pas encore de penser le comment de l'apparaître, précisément parce qu'il le réduit de manière précipitée à celui d'un objet pour un sujet.

Il revient alors à Heidegger de prendre pour thème la question de l'apparaitre comme tel sans lui imposer une orientation strictement objectivante (MARION, 2013, p. 6 et 55). Cette démarche est possible, parce qu'Heidegger ne prédétermine pas ce qui apparait, se limitant à reconnaitre qu'il y a ce qui est. Cet avoir-lieu n'est que dans la mesure où il se donne à un existant (le Dasein) qui est en question lui-même lorsque se joue cette donation. Il est donné à l'étant la possibilité d'apparaître par la médiation du Dasein qui le manifeste pour ce qu'il est à travers son projet de compréhension. La spécificité du Dasein, au regard des autres étants, provient de son pouvoir de les manifester et de se manifester (à) lui-même en les manifestant. Son mode d'être n'est pas étroitement ontique puisqu'il a le privilège de formuler la question de l'être des étants. C'est donc en vertu de cette différence ontologique qu'Heidegger aborde la question de l'apparaître de ce qui se donne. Or cette perspective conduit Marion à mettre en question la radicalité du projet d'Heidegger. A-t-on bel et bien accès à l'apparaître comme tel, à sa donation originaire, lorsque celui-ci est par avance pensé dans l'horizon de l'apparaître de l'étant et depuis la perspective du Dasein?

On peut faire valoir, en premier lieu, le caractère problématique de devoir rendre compte de la différence ontologique, comme source de 
l'apparaître, depuis le différencié, c'est-à-dire depuis le point de vue de l'étant Dasein qui enregistre le fait du déjà-là de l'étant et remonte, après coup pourrait-on dire, à sa condition de possibilité ontologique, l'être. N'apparaît en effet que ce qui est pour cet étant qui a le privilège de se poser la question de la manifestation de l'être. Autant dire alors que la question de l'être à partir de laquelle est pensée la question de l'apparaître se réduit à celle d'un étant particulier, celle du Dasein. Au lieu de penser la donation du phénomène par soi-même et comme tel, au lieu de prendre en charge la question de l'être, depuis la différenciation ou le se différenciant, le Heidegger de Etre et temps reconduit l'être à une condition de possibilité qui en restreint l'apparition, à savoir le Dasein (MARION, 2013, p. 56-59). Voici pourquoi, en second lieu, Heidegger sera conduit à réaliser la Kehre. Au lieu de penser la donation depuis le déjà donné à un étant-Dasein, il s'agira de comprendre la donation même de l'étant à un Dasein. Il ne s'agira plus de remonter du donné à sa condition de possibilité ontologique, le Dasein qui fait l'expérience de la différence ontologique, mais de partir de la différence ontologique, de l'advenir de l'étant à un Dasein à partir de l'être. Pour autant, ici encore la description de l'apparaître semble indûment limitée, dans la mesure où ne mérite description que ce qui se donne comme un étant à un existant. Or il n'est pas légitime, selon Marion, d'interpréter a priori tout procès de manifestation sous la figure du don de la présence (MARION, 2013, p. 59-67)². La phénoménologie ne doit-elle pas s'émanciper de l'ontologie si elle veut pouvoir rendre compte de la manifestation, non pas tant d'ailleurs pour donner congé à l'ontologie que pour en rendre compte depuis une instance véritablement originaire qui se trouve en-deçà de la différence ontologique?

Tel est précisément le projet de Jean-Luc Marion, puisqu’il s’agit de décrire la venue de la manifestation à la visibilité, son advenue au monde pourrait-on dire, son entrée dans le champ de ce qui est montrable par nous et pour nous au sein d'horizons. Il s'agit de décrire la manifestation en son procès, dans la dynamique de son déploiement, indépendamment de son être-advenu sous la

\footnotetext{
${ }^{2}$ Déjà dans le dernier chapitre de Réduction et Donation, J.-L. Marion montre comment Heidegger, qui avait la prétention de penser l'être à partir du rien tel qu'il s'éprouve dans l'angoisse est obligé de supposer une interprétation qui ne peut avoir pour guide que cela à quoi elle veut pourtant dégager un accès : le phénomène d'être. Faute de parvenir à penser un accès direct du rien à l'être, Heidegger installera la notion d'appel de l'être qui nous convoque à un destin ontologique. Mais dans la mesure où ce destin peut se refuser - comme l'atteste la tonalité de l'ennui profond - et où rien n'atteste que l'appel soit celui de l'être, il faut mettre en doute cette compréhension ontologique de la donation.
} 
forme d'un objet ou d'un étant ${ }^{3}$. La description de la donation consiste à rendre compte de la différence en vertu de laquelle la manifestation comme telle se fait phénomène pour nous. Or l'exigence philosophique ainsi formulée donne naturellement lieu au problème suivant. Est-il possible de remonter en deçà de la clause transcendantale (sujet intentionnel husserlien ou Dasein heideggerien), en vertu de laquelle tout apparaitre est toujours apparaître pour quelqu'un, sans renouer avec une forme naïve de réalisme dogmatique prétendant connaître la chose en soi et faisant fi de notre finitude? Existe-t-il un moyen de régresser en deçà de cette clause pour en montrer le caractère dérivé, sans pourtant l'abolir si l'on veut pouvoir rendre compte de la manifestation pour nous? Peut-on remonter en deçà de la corrélation husserlienne ou de la transcendance du Dasein non pas pour nier leur prétention à conditionner l'apparaitre pour nous mais afin de justifier, tout en le relativisant, leur pouvoir? Y-a-t-il un sens à décrire une manifestation en soi sans succomber à une vaine spéculation? Peut-on, au sein du donné, décrire le mouvement par lequel se donne les objets, les étants, tout phénomène en général?

Or, telle est bien la question que semble prendre en charge la phénoménologie de la vie de Renaud Barbaras. En effet, si l'on ne comprend pas l'apparaître du sujet (en son sens husserlien ou heideggerien) depuis le plan de l'apparaître en soi, on continuera à en avoir une conception métaphysique interdisant la description de l'apparaître en soi, préjugé d'autant plus dangereux que cette conception se présente par ailleurs comme la condition de la constitution de l'apparaitre. Que l'apparaître se donne à un sujet, que le sujet le co-conditionne ne signifie pas qu'il soit constitué par le sujet. C'est donc un impératif phénoménologique que de revenir au plan de l'apparaitre originaire, nécessairement en excès au regard de l'apparaître subjectif (ce pour quoi il y a une dynamique propre de la manifestation), pour pouvoir décrire ensuite cet apparaître de second degré. C'est dans ce qui se donne de surcroît que se trouve la clef de compréhension de la générosité phénoménale en vertu de laquelle quelque chose, toujours, se passe.

On peut donc rapprocher le point de vue de Barbaras de celui de Marion dans la mesure où tous deux dénoncent les limites de la phénoménologie traditionnelle lorsqu'elle prétend penser la donation à partir d'une conception transcendantale du sujet. Certes Barbaras prend pour point de départ l'a priori universel de corrélation mais dénonce cette démarche qui conduit à séparer la

${ }^{3}$ C'est ainsi par exemple que la contemplation esthétique d'un tableau ne peut délivrer son sens propre si l'œuvre est comprise sous la figure d'un simple objet, d'un étant usuel ou subsistant ou dans l'orbe de la vérité de l'être (MARION, 2013, p. 68-81), (CIOCAN, VASILIU, 2016, p. 367). 
conscience du monde faute de penser le mode d'être univoque qui permette de conjoindre la double dimension d'appartenance et d'ouverture du sujet. Il est vrai que Heidegger prétend décrire, dans son analytique existentiale, la manière dont le sujet peut se projeter vers le monde tout en lui appartenant. Néanmoins cette description reste formelle faute de prendre en compte la chair par laquelle le sujet appartient effectivement au monde. C'est pour cette raison que la question du vivre devient centrale chez Barbaras. Il souhaite montrer comment la vie provient du plan mondain, comment l'humain procède du vivant, ce qui impose de penser, au sein du mondain, une certaine "vie" (référence effacée), une puissance phénoménalisante permettant de comprendre la présence de ce même pouvoir au sein des vivants et du vivant humain en particulier. Cela signifie qu'il faut trouver l'origine du paraitre en deçà de la subjectivité et que la démarche doit donc être ontologicocosmologique, en ceci que l'essentiel de la réflexion se concentre sur l'être de l'intentionnalité en tant qu'il implique une compréhension du monde comme source originaire de la subjectivité phénoménologique $e^{4}$.

On pourrait alors avoir l'impression ici que, même si cette démarche se caractérise par une certaine concrétude, puisqu'en s'interrogeant sur le vivre du sujet elle ne fait pas qu'énoncer le fait de l'inscription du sujet dans le monde mais cherche à la justifier à partir des choses mêmes, elle reste cependant dépendante d'une pensée de la différence ontologique, qui interdit de comprendre l'apparaître à partir de lui-même, comme c'est le cas chez Marion, limite préjudicielle d'autant plus problématique qu'elle aborde la différence depuis le différencié, à savoir le sujet phénoménologique, qui, fût-il charnel, n'en reste pas moins la charnière transcendantale à partir de laquelle Barbaras prétend remonter à son origine, loin qu'il ne parte de cette origine comme différence originaire.

Néanmoins, Barbaras ne prétend pas penser la différence ontologique depuis le différencié. Il s'agit au contraire pour lui de remonter en deçà de cette différence et d'en produire la genèse. Cela ne signifie pas seulement qu'il examine la différence ontologique depuis elle-même mais plutôt qu'il veut en produire la genèse, en examiner le principe de production. Comme le note Barbaras (2013, p. 205):

\footnotetext{
${ }^{4}$ Les idées de ces deux derniers paragraphes ont été exprimées dans "Archi-acontecimiento y carne en el pensamiento de Renaud Barbaras" in "Los limites de la fenomenicidad", Escritos de Filosofia, Academia Nacional de Ciencas de Buenos Aires nº (2018)
} 
[...] la différence ontologique apparaît comme dérivée d'une différence où il n'y a pas encore de l'être ou de l'étant mais un fond indifférencié - que l'on peut appeler être par une sorte de passage à la limite et d'hypostase - et un champ différencié que l'on a identifié à celui des étants, mais à la condition de comprendre que leur étantité est dérivée de la différenciation au sein de ce champ mondifié.

Et s'il est incontestable que Barbaras (2013, p. 110) voudra établir "[...] une ontologie universelle de la vie", c'est en un sens bien particulier de l'ontologie qu'il faut entendre le sens de sa démarche, dans la mesure où l'être ne sera pas l'a priori de la question de l'apparaître sinon bel et bien la manière de la spécifier. C'est en comprenant pour elle-même comment la manifestation a lieu et se fait monstration pour un sujet que l'on est amené à poser la question ontologique. La différence phénoménologique conduit à la différence ontologique, loin que ce soit l'inverse. De ce point de vue, Barbaras, tout comme Marion, prétend s'affranchir d'une inféodation à la manière que le second Heidegger a de poser la question de l'apparaître.

C'est, en tout état de cause, ce qui nous semble se dégager d'un précieux article de Barbaras consacré à Patočka (BARBARAS, 2007, p. 29-43) et qui nous semble valoir pour sa propre philosophie, dans la mesure où elle s'inspire de la démarche du philosophe tchèque. En effet, après avoir rappelé que Patočka rend hommage à Heidegger pour penser l'être comme dévoilement, c'est-àdire comme manifestation irréductible à l'instance subjective de constitution du phénomène, il faut néanmoins remarquer que loin que l'apparaitre soit le thème de la phénoménologie heideggerienne, c'est l'être - comme concept abstrait- qui s'y substitue, et la phénoménologie, devenue simple méthode, se met alors au service de ce concept. En séparant la méthode phénoménologique de son thème propre, l'apparaître, Heidegger s'interdit de comprendre l'être selon l'apparaître. C'est d'ailleurs parce que la question de l'être est abordée préalablement à celle de l'apparaître que Heidegger est conduit à faire assumer l'apparaître à une instance subjective - le Dasein - au lieu de penser l'être depuis son apparaître. Il ne s'agit pas de dire que, comme Husserl, Heidegger subjectivise l'apparaître parce qu'il le réfère d'emblée à un apparaissant singulier, mais plutôt parce qu'il pose la question de l'être prématurément, abstraitement, antérieurement à la question de l'apparaître ce qui l'oblige à

\footnotetext{
5 De ce point de vue, la théorie de l'indication formelle comme méthode visant à ne pas prédéterminer ce qu'il y aurait, à savoir de l'être, ne fait que corroborer la suspicion à l'égard d'une démarche méthodologique qui tente de prendre en charge un thème défini hors phénoménologie (l'être) et qui,
} 
poser une instance subjective de manifestation de l'être, au lieu de comprendre le sujet comme un moment même de l'apparaître (BARBARAS, 2007, p. 36).

Il faut donc affirmer un primat de l'apparaître comme préalable à la question du sens de l'être. Force est alors de reconnaître que ne paraît que ce qui se différencie des autres choses et qui, se faisant, s'affirme comme étant la même chose, fait valoir Barbaras toujours dans le cadre de son commentaire de la pensée de Patočka. Elle n'est donc elle-même que pour autant qu'elle se détache d'une totalité auquel elle reste cependant liée, ce qui signifie que la structure primordiale de l'apparaître est le monde, entendu comme l'ensemble de tout cela qui peut apparaître. La chose n'est qu'autant qu'elle paraît en se différenciant d'une totalité avec laquelle elle est en rapport, le monde. C'est donc comme monde qu'il faut envisager l'être compris depuis l'apparaître en tant qu'il est le tout des apparitions possibles qui implique, comme lui étant inhérent, une dimension de voilement, puisqu'il excède toujours, en tant que tout, toute apparition particulière. C'est parce que tout apparaître est apparaître d'un monde qu'il suppose un recouvrement. Ce n'est donc pas l'être qui est voilé, pour ainsi dire en soi. Il faut plutôt dire que l'apparaître, en vertu de sa structure propre, implique un voilement. Comme l'écrit Barbaras (2007, p. 41): "L'être en tant que tel, comme différant de la manifestation, n'est rien d'autre que le voilement même de l'apparaître et ce voilement est constitutif de l'apparaître en tant qu'il est apparaître d'un monde". La différence ontologique de l'être au regard de l'étant, le voilement inhérent à la manifestation de l'étant, est donc subordonnée à la différence phénoménologique au sens où l'apparaissant différencié suppose un apparaître-un se différenciant. L'essence de la manifestation réside dans une transcendance de cet apparaitre au regard des apparaissants finis qui en sont la limitation mais également l'expression incessamment renouvelée. En dépit même des réserves de Barbaras à l'égard de la pensée de Patočka, en particulier pour ce qui touche aux questions de l'émergence de la vie et de la différence anthropologique (BARBARAS, 2007, p. 112 note 1), il nous semble que Barbaras assume, comme lui et tout comme Marion, le projet de penser une différence phénoménologique en-deçà de la différence ontologique telle que la conçoit Heidegger. Il s'agit de produire la genèse de cette différence à partir de la question de l'apparaître comme tel. C'est pour cette raison que Barbaras (2007) pensera la différence ontologique, l'apparaître de l'être en tant qu'il implique une occultation, comme délimitation, détachement ou séparation

se faisant, se désolidarise de la prétention à décrire concrètement la phénoménalité. (FRANCK, 2001, p. 154-155). 
depuis une unité préalable (celle du monde) plutôt que comme dévoilement. Il s'agit en effet d'éviter le risque de subjectivisation qu'encourt la pensée du dévoilement, puisque celle-ci présuppose une instance subjective en charge de dévoiler ce qui est recouvert. L'hénologie est alors l'autre nom de l'unité phénoménale, qui précède la différence ontologique, et rend compte de la manifestation comme transcendance, comme différence dynamique.

Si donc on trouve chez Barbaras une pensée du vivre et une présence de l'ontologie qui, de prime abord, semblent plutôt atténuées chez Marion, il n'en reste pas moins que les deux auteurs semblent avoir ceci de commun qu'ils prétendent rendre compte de la différence phénoménologique pour ellemême, en-deçà des différences épistémologiques et ontologiques qui lui sont dérivées. L'essence de la manifestation parait faire signe vers un excès, à partir duquel on peut comprendre la dynamique d'une phénoménalité dont le sujet peut faire l'épreuve lorsque tel ou tel phénomène se donne à lui.

Mais avant même de décrire comment ces phénomènes adviennent au sujet et ce que doit être le sujet pour pouvoir accueillir cette phénoménalité, il convient d'indiquer brièvement quelle méthode est à même, selon Jean-Luc Marion et Renaud Barbaras, de décrire directement, de manière "asubjectiviste", l'apparaître. Que doit être la méthode de la phénoménologie pour qu'elle soit à la hauteur de son thème le plus propre, l'apparaître comme tel?

\section{RECONDUIRE À LA SUSPENSION}

Cela revient à se demander comment le sujet peut se déprendre suffisamment de lui-même (de sa tendance à objectiver et chosifier, à expliquer et comprendre) pour remonter à la source de l'apparaître à partir de laquelle non seulement les phénomènes se donnent par eux-mêmes mais donnent aussi au sujet la possibilité de s'apparaitre à lui-même, non comme cela qui constitue le phénomène (subjectivité intentionnelle ou Dasein) mais comme cela qui en témoigne, en atteste ou le recueille. Comment ce sujet peut-il donc s'effacer pour qu'apparaisse le phénomène comme tel tout en "existant" suffisamment pour le recevoir? Comment conjuguer la passivité d'une réception aussi neutre que possible, qui prend le risque de ne plus même pouvoir témoigner du phénomène, et l'indispensable activité minimale qui permet l'accueil phénoménologique, au risque de l'abolir en en faisant une simple représentation? 
Il semble néanmoins qu'il n'y ait là un problème de méthode qu'aussi longtemps qu'on considère cette dernière comme prioritaire au regard, et indépendante, du thème phénoménologique qu'elle doit permettre de porter au concept. Elle devient dès lors une procédure ou une attitude qui requiert, de la part du sujet, une démarche préalable et la mise en ouvre de recommandations ou de règles de caractère formel afin de pouvoir recueillir le phénomène. Mais, ce faisant, c'est bien alors, un risque de subjectivisation du phénomène à décrire que l'on court, puisqu'au lieu de comprendre le phénomène à partir de lui-même et de penser le sujet selon le phénomène tel qu'il se donne, on décrit le phénomène depuis une procédure mise en œuvre par un sujet conçu a priori comme porteur et initiateur d'une méthode qui vient de lui. Par ailleurs, c'est à la tentation d'utiliser cette méthode pour accréditer la valeur phénoménologique du thème choisi, comme si quasi tout thème pouvait devenir phénoménologique par la grâce de la méthode, à laquelle on s'expose. En aliénant le phénomène de lui-même au profit de sa validation méthodologique par une instance subjective pré-définie, on subjectivise cela qui pouvait mériter, par soi, le titre de phénomène et on se détourne de la description de son apparaître comme tel.

De ce point de vue, remettre en cause le primat de la méthode permettrait de lever le conflit entre la nécessaire activité du sujet pour constituer le phénomène et son indispensable passivité pour le recueillir selon la dimension a-subjective, ou plutôt pré-subjective de son apparaitre (puisque tout phénomène qui se donne se montre à un sujet). En restituant à la phénoménologie sa priorité thématique et en reconnaissant qu'elle a pour vocation première de porter au thème l'apparaître comme tel, on ne prédétermine pas sa méthode (qui épouse le phénomène et peut d'ailleurs varier selon) et la conception du sujet procède de cette description phénoménale au lieu de la précéder. L'effort de méthode ne consistera donc ici qu’à neutraliser tout ce qui du sujet pourrait compromettre la pureté de la description phénoménale pour s'en tenir au phénomène tel qu'il se donne.

C'est bien sur ce point encore que Barbaras et Marion semblent se rejoindre. Dans les deux phénoménologies en effet, on constate un effort pour neutraliser toute tendance appropriante de la part du sujet qui pourrait le conduire à prendre possession du phénomène au lieu de prendre acte de sa survenue.

De ce point de vue, il n'y a pas, aux yeux de Marion, un conflit entre réduction et donation (ROMANO, 2000; MARION), comme si la réduction 
supposait une initiative de la part du sujet constituant, contradictoire avec le surgissement simple du phénomène qui ne requiert aucune méthode pour se donner. C'est qu'en effet il faut bien une méthode pour s'en tenir au seul phénomène, pour se dépouiller de la "pourpre transcendantalice" (MARION, 2001, p. 59), et revenir à l'immédiat. On aurait donc tort de confondre l'initial et l'immédiat. Ce dernier se prépare par une étonnante pédagogie qui enseigne l'abstention et invite à la suspension. S'il est vrai que l'advenir du phénomène est cela qui ne se prépare pas au sens de l'acquisition d'un savoir-faire, et peut-être aussi cela qui ne se vit pas (sous forme d'un vécu de conscience ou d'un projet de compréhension, puisqu'il les déborde, les défait ou les reconfigure), il est sans doute aussi cela à quoi on peut se rendre disponible, par une forme de déprise de désobjetivation ou de déréification. Se rendre ouvert à cela qui se donne comme il se donne requiert de se défaire des expériences d'appropriation, en ce que celles-ci ne sont pas des épreuves de l'altérité phénoménale sinon des répétitions du même. Voici pourquoi une méthode (paradoxale) est requise pour se préparer à ce qui ne se prépare pas, en renonçant aux formes préconstituées de l'expérience et en suivant comme unique principe celui de renoncer à tout principe a priori afin de construire ses catégories au fil de l'expérience phénoménale. C'est bien en ce sens que Marion considère que le principe de la donation est un principe de réduction a posteriori qui se limite à laisser venir le phénomène pour le laisser se donner sans le pré-constituer (MARION, 2013, p. 30). Ce principe de méthode procède du thème même qu'il doit porter au concept, l'apparaître en ce qu'il se donne par lui-même sans que rien ne le précède. C'est seulement avec un tel concept de réduction, selon Marion, que la phénoménologie pourra prendre "l'initiative de la perdre" (MARION, 2013, p. 13).

Cette perte d'initiative qui permet d'accéder à l'apparaître comme tel, c'est-à-dire à son mouvement de venue à la visibilité pour nous, à sa monstration, se déroule en deux temps. En premier lieu, ce qu'il faut réduire, c'est un phénomène tout à fait singulier. De fait, le mouvement d'apparaitre procède d'une donation qui, pour échapper à l'abstraction, doit pouvoir se lire dans un phénomène particulier, sans que celui-ci le soit au point de ne nous renseigner que sur lui-même, puisqu'il doit, au contraire, ouvrir à la compréhension du procès de la phénoménalité en général. Quel est donc le singulier phénomène qui conserve en son sein la trace de sa venue à la visibilité? Comme l'écrit en effet Marion (2013, p. 520): 
[...] il garde en effet toujours, au terme de ce dépli, la marque du passage, du trajet ou du mouvement qu'il a accompli pour advenir ; le donné atteste par le tremblement dont il vibre encore et toujours, non seulement sa différence irréversible et répétée. Il atteste donc que s’il apparaît (se montre), il ne le doit qu'à soi, qu’à son soi (qui se donne).

Ce phénomène privilégié qui donne à voir la dynamique de la manifestation en général, c'est le don. Il ne s'agit pas là d'anthropologiser, voire de théologiser, la phénoménologie en excédant indûment les limites d'une simple analogie entre une relation dont les termes sont humains (le donataire, le don, le donateur) et celle dont les termes sont les phénomènes donnés et le sujet qui les reçoit. La rigueur de Marion consiste bien plutôt à tirer les conséquences, après Derrida, du caractère aporétique (MARION, 2013, livre II) de l'apparaitre du don. Puisqu'on ne peut en rendre compte selon les lois ordinaires de l'apparaître, c'est-à-dire selon la logique intuitionniste de Husserl, force est de reconsidérer ces lois pour que puisse apparaître le don. En d'autres termes, c'est depuis la phénoménologie de la donation, vers laquelle les impasses de la phénoménologie traditionnelle du don nous obligent à nous tourner, que le don pourra se montrer, mais c'est tout aussi bien dire que c'est à partir de lui, de la description du fait de donner, qu'on pourra apercevoir le mouvement en vertu duquel ce phénomène apparaît ${ }^{6}$. En lui se donne donc non seulement le donner de tout don mais également la venue à la visibilité du phénomène en général, puisque le don n'est après tout, et en ce sens, qu'un phénomène parmi d'autres. Comment d'ailleurs pourrait-on même continuer d'affirmer son existence si l'expérience de cet impossible n'invitait pas à réordonner les conditions de sa manifestation grâce à un renouvellement des coordonnées de la phénoménologie au prisme de la donation??

Mais ce premier pas réductif, qui reconduit du don à sa donation à un sujet (au lieu d'être constitué par lui) et ouvre à une pensée de l'apparaître comme cela qui se donne à nous, exige d'effectuer un pas supplémentaire. La reconduction à la suspension comme tel (de l'empirique) - c'est-à-dire, au maintien dans le suspensif, puisque la réduction ne reconduit pas à une conscience constituante ou à un Dasein compréhensif mais à la donation comme dynamique de la manifestation - exige également de prendre acte

\footnotetext{
${ }^{6}$ Dans (MARION, 2013, livre III), l'auteur montrera comment les propriétés du donnés s'appliquent, reçoivent un équivalent au plan de la phénoménalité, dans la mesure où tout phénomène est donné.

${ }^{7}$ Contre Derrida, qui s'en tient à l'expérience d'un impossible, d'une aporie, qui n'immobilise pas mais renvoie à un excès de ce qui se donne sur la compréhension.
} 
du caractère gradué de cette donation. Il ne suffit pas de découvrir que tout phénomène est un donné qui reconduit à une donation, de déplier le pli de la donation. Il faut aussi reconnaitre que cette donation se donne plus ou moins à éprouver selon le phénomène considéré. L'évidence de la donation, pour qui n'est pas prisonnier de la rigidité métaphysique qui définit a priori les limites de l'appréhendable, est plus ou moins forte selon que le phénomène semble venir de lui-même ou bien "de" nous, selon qu'il se présente à nous comme cela qui excède notre pouvoir de donner sens ou au contraire comme cela qui nous donne à croire que nous en sommes la source. La thèse selon laquelle il existe des degrés de donation permet à Marion d'unifier le champ du phénoménal - tout se donne (objet, étant, phénomène saturé...) même si cela ne se donne pas selon le même degré - et de faire signe vers la source d'une illusion philosophique: c'est parce que l'on a donné la priorité aux phénomènes pauvres en intuition qu'on a pu exclure les phénomènes saturés et croire au caractère constituant du sujet. La réduction bien comprise est, comme nous l'avons dit, ce principe de méthode qui laisse le phénomène apparaître sans l'entraver a priori par une limite (celle du prévisible, de l'horizon, du sujet.) Cette réduction est donc elle-même susceptible d'intensité, selon que le sujet se met à la hauteur ou non de l'intensité du phénomène considéré, selon donc par exemple qu'il atteste de l'événementialité du phénomène saturé en le vivant, en reconfigurant ses schèmes interprétatifs à la lumière de ce qui se donne. Ce n'est plus le sujet qui éclaire le phénomène, c'est au contraire le phénomène qui lui permet de s'éclairer, de se comprendre comme cela qui peut l'accueillir, comme attributaire ou adonné.

Ainsi présenté on pourrait dire que Barbaras est assez proche d'un tel choix méthodologique, subordonné à l'enjeu thématique de la phénoménologie: décrire l'apparaître comme tel. De fait, pour Barbaras également, il s’agit de procéder à une réduction en tant qu' elle permet d'accéder au champ de l'apparaître en soi. Cependant, même s'il s'agira de penser cette réduction en tant qu'elle permet au phénomène de se donner comme tel sans l'enfermer par avance dans des règles a priori et subjectives d'appréhension, il s'agira moins pour lui de s'appuyer sur un phénomène particulier (le don ou même tel ou tel phénomène saturé) en tant qu'il défait le cadre traditionnel et subjectivisant de la phénoménologie que de chercher à la racine, à travers une destruction de l'histoire de la phénoménologie, le principe de cette subjectivisation pour le neutraliser et ouvrir ainsi, à nouveaux frais, la voie d'accès au champ de l'apparaître comme tel. Tel est bien le sens de l'épochè sur la mort réalisé dans l'Introduction à une phénoménologie de la vie (BARBARAS, 
2008). En montrant que la tendance à comprendre l'homme, comme principe de l'apparaître, provenait d'un préjugé obscur de la philosophie qui se le représente comme conscience s'ajoutant à la vie, interprétée comme simple activité d'auto-conservation en conséquence du péril de mort qui la menace, Barbaras nous invite à suspendre cette conception "réaliste" de la vie pour la décrire selon elle-même, indépendamment de cela qui lui est extérieur et prétend la menacer, la mort. En réalisant une épochè sur la mort, on ne niera certes pas l'existence de ce phénomène - puisqu'on pourra au contraire en comprendre le sens véritable depuis la vie - mais on rendra possible une interprétation de la vie à partir de laquelle il est possible de saisir en quel sens le sujet humain se fait pouvoir de monstration du monde. La vie n'est pas cela qui s'oppose à la conscience phénoménalisante comme son autre, mais au contraire mouvement de visibilisation qui peut donner lieu à la conscience intentionnelle humaine (Référence effacée). Cette réduction a un sens original, puisqu'il s'agit d'une épochè qui ne reconduit pas à la conscience humaine mais à la vie. Celle-ci n'étant plus activité aveugle d'auto conservation rend possible l'activité humaine phénoménalisante. C'est donc une réduction qui ouvre au champ de l'apparaître comme tel, à l'Ouvert comme écrit Barbaras, en tant qu'il se donne à la vie et non à la conscience. Si l'on suit la lecture d'Emilie Tardivel (TARDIVEL, 2015) qui interprète la réduction opérée par Marion dans le sens de Patočka qui procède à une épochè sans réduction à la conscience (PATOČKA, 2002, p. 217-228) afin de décrire l'apparaître selon ses lois propres (qui, pour le phénoménologue tchèque, sont celles du monde), force est de reconnaître une certaine proximité entre l'épochè sur la mort de Barbaras et la réduction à la donation de Marion. Toutes deux permettent en effet de suspendre non seulement l'attitude naturelle en vertu de laquelle nous pensons être dans un monde déjà là mais également la croyance en vertu de laquelle c'est une instance subjective qui se donne l'apparaitre. Toutes deux, mais chacune à sa façon, créent ainsi les conditions pour penser non seulement la venue à la manifestation mais également l'émergence de la subjectivité depuis cet apparaître originaire.

\section{L'ÉVÈNEMENT DE L'APPARAîTRE ET LE SURGISSEMENT DE LA SUBJECTIVITÉ}

Avant de commencer à prendre la mesure de ce qui peut éloigner les deux phénoménologues de l'apparaître, il importe d'insister sur la communauté de perspective quant à la description de l'apparaître comme événement. 
La réduction à la donation permet en effet de saisir la manifestation comme cela qui arrive au sujet. Le phénomène, ne pouvant plus être constitué par les catégories subjectives a priori, est reçu. On pourra toujours se demander si ce n'est pas là une expérience impossible, puisqu'elle transcende les conditions de possibilité de l'expérience et de la connaissance d'un objet. Mais il faudra plutôt reconnaître que cette "contre-expérience" est en vérité la seule expérience authentique dont nous soyons capables, puisqu'au lieu d'imposer à ce qui se donne cela qui vient du sujet (les formes sensibles et les catégories, si l'on suit Kant) elle le met effectivement à l'épreuve de ce qui n'est pas lui. Il faut alors bien appeler évènement cela qui ne peut s'attester que dans son advenue, puisque le phénomène devient inanticipable, irréductible au principe de causalité (MARION, 2010; 2013) ${ }^{8}$.

Mais ce n'est pas simplement l'advenue de la manifestation qui est un événement pour le sujet, c'est également ce sujet qui devient évènement pour lui-même lorsqu'il montre cela qui se donne à lui. On peut certes passer sous silence cette dimension évenementiale de la subjectivité lorsque les phénomènes considérés sont pauvres, au point de laisser accroire qu'un sujet les constitue, mais puisque c'est le phénomène saturé qui est la vérité du phénomène parce qu'en lui se donne à voir, avec un certain éclat, la trace de la donation, il n'est plus possible pour le sujet averti de s'ignorer lui-même, comme témoin de la manifestation. C'est à l'occasion de l'évènement de l'apparaître qu'il découvre, sans avoir pu l'anticiper, ce qu'il en est de lui-même, cela qui se reçoit de recevoir le phénomène, comme dit Marion.

Or l'épochè sur la mort permet, selon Barbaras, de revenir en amont de la conscience intentionnelle tournée vers le monde pour rejoindre l'apparaitre depuis un vivre qui s'y destine. Mais si la conscience humaine qui vise un monde procède d'une telle ouverture primordiale, il faut comprendre en quel sens celle-là procède de la négation de celle-ci. Puisque l'homme ne peut plus être une conscience ajoutée à la vie, sauf à retomber dans un dualisme qui interdit de saisir l'origine de l'a priori universel de corrélation, mais bien plutôt une conscience en tant qu'elle résulte de ce qu'il faut ôter à la vie $^{9}$ conçue comme puissance intrinsèque de visibilisation, pour qu'elle ne soit plus que conscience du monde, il reste à comprendre comment cette

\footnotetext{
8 Sur l'évènement, voir (MARION, 2013, $\$ 17$; MARION, 2001, chapitre II ; MARION, 2010, chapitre V). Il n'est pas seulement ce qui excède la catégorie de la quantité et permet de décrire un certain type de phénomène saturé. Il est aussi ce qui permet de comprendre la phénoménalité en général.
}

${ }_{9}$ C'est ce que Barbaras nomme l'anthropologie privative. 
soustraction peut ne pas donner lieu à la restauration ou bien d'un dualisme -si elle se fait différence substantielle- ou bien d'une perte de la spécificité de la différence anthropologique dans l'homogénéité de la vie, si cette soustraction est purement quantitative. Si la conscience humaine surgit de ce que le mouvement vers l'infini de l'Ouvert est tout aussi bien refoulement de ce mouvement, dès lors que tout avancée vers l'infini est tout aussi bien recul devant lui, comment penser ensemble la différence humaine à l'égard de la vie sans perdre sa continuité avec elle? Si la différence humaine procède d'un certain type de refoulement de la vie, il faut alors comprendre comment la vie est, de part en part, auto-refoulement et en quoi consiste la spécificité humaine à cet égard. Chez Barbaras également il s'agit donc de comprendre le surgissement de la subjectivité humaine à partir d'une manifestation originaire qui met en jeu l'Ouvert pour le vivre.

On pourrait ajouter que cette question met en jeu, ici aussi, une pensée de l'événementialité. L'apparaître obéit en effet à une structure événementiale tout comme le surgissement de la subjectivité. Mais pour clarifier cet aspect des choses, il faut se tourner vers Dynamique de la manifestation puisque le but de cet ouvrage, de l'aveu même de Barbaras (2013, p. 336), est de "[...] répondre à la question de la possibilité de cette auto-négation, de se donner les moyens théoriques permettant de généraliser l'anthropologie privative sous la forme d'une zoologie privative", et donc de répondre à la question précédemment soulevée. L'auteur parvient effectivement à proposer une "ontologie universelle de la vie" (BARBARAS, 2013, p. 110) en comprenant le monde comme procès de manifestation à partir duquel on peut penser le surgissement du sujet en cohérence avec son mode d'être. Pour autant, que cette conception du monde n'interdise plus de voir advenir une subjectivité en son sein ne signifie pas encore qu'on sache comment il se fait qu'elle advienne effectivement (BARBARAS, 2016, p. 43-45). Autant dire alors qu'on ne peut en rester au plan d'une compréhension cosmologique pour rendre compte de cet avènement. Le monde est mobilité pure, procès de différenciation des étants à l'égard du tout. Puisqu'il ne s'achève jamais, l'être est toujours en retrait au regard des étants manifestés, toujours voilé. Mais il ne peut se manifester autrement que sous la forme de cette délimitation incessante des étants à l'égard de l'ensemble et, en ce sens, il est fini. Comme nous l'avons indiqué, cette finitude ontologique, comprise à partir des conditions propres de l'apparaître, explique la production et la manifestation des étants. Comme nous le disions également, l'avènement du sujet depuis ce plan primordial de l'apparaître (mondain) devient donc possible. Cependant, s'il est désormais clair que des étants puissent paraitre depuis le monde, on ne 
comprend pas encore pourquoi ce type d'étant qu'est la subjectivité puisse cesser de se réaliser sous la forme d'une production afin de montrer, pour son compte propre, le monde.

Si le monde est réellement pur mouvement, comment expliquer que l'étant-sujet puisse renoncer à accomplir ce mouvement de réalisationproduction pour faire paraître le monde et se faire paraître à elle-même? C'est la formulation de cette question qui conduit Barbaras à formuler l'hypothèse d'un archi-fait qui affecte le monde de manière à produire, en son sein, une scission grâce à laquelle est interrompue ou affaiblie cette mobilité. Ni cause ni raison ne permettent de justifier cela qui apparait donc comme un événement. Et c'est de cet archi-évènement que procède la subjectivité vivante. Pour maintenir une différence entre l'animalité et l'humanité sans perdre leur continuité, l'auteur pense une telle différence en termes de tension ou de polarité. En effet, l'animal tend à rester sous l'empire de l'archi-mouvement tandis que l'homme, pour sa part, est davantage affecté par les effets de l'archiévènement. C'est pour cette raison qu'il ne peut rejoindre le monde, auquel il reste attaché, que par le biais de la phénoménalisation, qui est ce qui du mouvement lui reste après que l'archi-évènement ait produit son effet. L'être subit donc une seconde finitisation, cette fois évènementiale et non plus simplement ontologique, grâce à laquelle adviennent les sujets vivants. Ils se séparent de la puissance originaire du monde en vertu d'un affaiblissement provoqué par l'archi-évènement. Ce surgissement du sujet provoque, de manière corrélative, une séparation des choses à l'égard du tissu ontologique : elles s'absentent du monde, perdent en "réalité " et c'est ainsi qu'advient leur sens. C'est bien pour cette raison que le sujet découvre le phénomène comme lui advenant et comme porteur d'une dynamique en vertu de laquelle ce sujet n'est plus producteur mais destinataire de l'apparaître. C'est pour cette raison aussi que le je-ne-sais-quoi de la différence, qui fait qu'au sein du phénomène on fait également l'épreuve de son advenue, est en dernière instance de caractère métaphysique. Comme l'écrit Barbaras (2016, p. 121):

[...] la différence entre vivants et non-vivants n'est pas organisationnelle ou chimique: elle est métaphysique. Plus, elle est la différence métaphysique même, la métaphysique comme différence, comme l'évènement de la différence.

Une telle conception de la différence a une double portée. D'une part, elle rend intelligible l'avènement du sujet phénoménologique depuis le plan 
du monde, en décrivant comment une négation par affaiblissement s'ajoute à une négation par délimitation. D'autre part, elle clarifie le statut de la différence anthropologique qui consiste en une différence de degré au sein du règne vivant. L'homme est plus "sensible " que l'animal à l'archi-évènement et c'est cette négativité métaphysique qui fait que l'être prend, à ses yeux, les traits d'une question ${ }^{10}$.

\section{Perspective ConClusive: Le PROBlÈME DE LA NAISSANCE DU SUJET ${ }^{11}$}

Cette présentation bien que sommaire des dispositifs respectifs de chacun des deux philosophes français suffit pour autant à indiquer certaines divergences qui feront signe vers le caractère central de la question de la naissance du sujet et tels seront les résultats proposés au sein de cet article. C'est en effet parce que Barbaras entreprend la description de l'apparaître comme tel depuis une cosmologie dynamique qu'il peut se poser la question non pas seulement de l'inscription et de l'émergence de la subjectivité depuis le monde mais également de son surgissement en tant que rien dans le monde ne peut expliquer la déchirure qu'il présuppose et dont elle est l'attestation. L'événement de la subjectivité procède d'un évènement qui arrive au monde et contraint Barbaras à développer une métaphysique de la facticité à laquelle Marion n'est pas conduit, non pas simplement parce que toute métaphysique ne pourrait être qu'ontique pour lui mais plutôt parce que sa description de la dynamique de l'apparaître - la donation comme mouvement de venue à la manifestation - n'est pas élaborée à partir d'une bio-cosmologie. De ce point de vue, Marion semble en rester à un plan strictement phénoménologique, sans prétendre pouvoir ou devoir effectuer une archéologie de la manifestation sous la forme d'une cosmo-métaphysique, apte à rendre compte du surgissement du sujet depuis un monde transi par des mouvements incessants de manifestation et affecté métaphysiquement par un événement provoquant la scission interne, favorisant une séparation - jamais radicale - à la faveur de laquelle a lieu la venue au monde du sujet phénoménologique.

\footnotetext{
${ }^{10}$ Les idées de ces trois derniers paragraphes ont été exprimés dans "Archi-acontecimiento y carne en el pensamiento de Renaud Barbaras" in "Los limites de la fenomenicidad", Escritos de Filosofia, Academia Nacional de Ciencas de Buenos Aires nº (2018).

${ }^{11}$ Le problème phénoménologique de la naissance est l'objet actuel d'une élaboration de caractère métaphysique par Frédéric Jacquet. Voir en particulier: JACQUET, F. Métaphysique de la naissance. Louvain-la-Neuve: Peeters, 2018.
} 
Faut-il alors considérer que, tandis que Marion s'en tient aux limites de la seule description phénoménale, Barbaras franchit un Rubicon qui l'empêche de trouver dans le registre des phénomènes une attestation quelconque non seulement de cet archi-événement, dès lors plus postulé que constaté, mais également de cet archi-mouvement qui assure la "branloire perenne" (MONTAIGNE, 1965, p. 350) du monde comme dirait Montaigne, ou bien que, tandis que Barbaras remonte à la source de la dynamique de la manifestation et peut rendre compte de l'événementialité de l'apparaitre, de ce je-ne-sais-quoi qui dans le phénomène n'est pas phénomène mais son advenir même, Marion court au contraire le risque d'une "déréalisation" de l'événementialité de l'apparaître, faute de penser les conditions biocosmologiques de cet apparaître?

Pour répondre à de telles préoccupations, il convient de faire deux séries de remarques. En premier lieu, s’il est vrai que l'archi-événement nous sépare de l'archi-mouvement du monde et fait ainsi naître la subjectivité en même temps qu'il rend possible la monstration du monde par le sujet, il faut se demander ce qui nous permet, au sein de l'expérience que nous avons, de remonter en-deçà de cet acte originaire grâce auquel nous sommes sujets phénoménalisant, pour attester de cet archi-mouvement dont nous venons. Pouvons-nous remonter en-deçà de notre propre finitude pour revenir au monde-patrie dont nous avons été exilés? Existe-t-il une expérience contreévènementiale de laquelle nous pouvons nous autoriser pour accréditer l'existence de cet archi-évènement qui nous a rendus possibles? C'est poser la question de la naissance du sujet comme sujet (BARBARAS, 2016, p. 197). En vérité, nous ne pourrions prendre conscience de notre finitude si nous ne l'avions pas déjà dépassée ou excédée. Nous ne pourrions pas nous savoir exilés si nous n'avions pas gardé trace de l'expérience originaire d'où l'on vient. Cela ne signifie pas pour autant que l'accès à l'originaire ou à l'absolu gomme ou supprime notre finitude. C'est bien de manière finie, à la façon d'un être fini, que nous dépassons notre finitude. La conscience de notre finitude est possible parce que nous l'excédons, mais l'épreuve même de ce dépassement nous rappelle à notre finitude. Or c'est dans le sentiment, selon Barbaras, que l'on se trouve au plus près de soi-même en même temps qu'on expérimente la pure ouverture qu'est le monde et le principe de son incessante mobilité. Je m'ouvre à moi-même parce que ma polarisation par le monde, le vide qu'il creuse en moi, me dépossède de tout contenu et non seulement m'ouvre à ce qui se donne comme il se donne mais également à moi-même comme "pure exposition à". Comme l'écrit l'auteur de Métaphysique du sentiment: "c'est en 
abdiquant de tout sens, de toute donation de sens, même simplement sensible qu'il peut accueillir la profondeur du monde, s'égaler à elle." (BARBARAS, 2016, p. 185). Et: “[dans le sentiment] nous n'éprouvons rigoureusement rien, si ce n'est précisément nous-mêmes en notre finitude et par conséquent aussi nous-mêmes comme aptitude à l'épreuve, être exposé à." (BARBARAS, 2016, p. 191) Cette passivité supérieure, indissociable du désir ${ }^{12}$, est cela qui précède mon rapport perceptif au monde, rendu possible par la scission originaire qui me sépare de la dynamique primordiale et rend possible l'objectivation. Ce sentir d'avant le sentir, "cette extimité existentielle" (BARBARAS, 2016, p. 193), ce sentiment du monde, et l'existence poétique qu'il rend possible, semble alors le radeau sur lequel nous pouvons prétendre rejoindre l'Ithaque perdue, et ainsi fuir de notre finitude (BARBARAS, 2016, p. 175).

En second lieu, si Marion ne décrit pas la manifestation en son dynamisme même depuis une origine cosmo-métaphysique, c'est plutôt qu'il y a là une option qu'il semble se refuser à prendre. D'où vient en fait l'exigence, chez Barbaras, de poser l'existence d'un archi-évènement si ce n'est de la nécessité de rendre compte de la manière dont le sujet vivant, et plus encore humain, doit perdre en mobilité et en activité pour gagner en réceptivité et ainsi être capable de montrer le monde, en particulier par le moyen de la perception? La monstration du monde est cela qui reste de pouvoir à un sujet qui a perdu contact avec l'archi-mouvement du monde et cherche pourtant à le rejoindre par des moyens propres. Une fois que l'archi-mouvement s'est imposé comme une nécessité pour rendre compte de l'être de "l'a priori universel de corrélation" (BARBARAS, 2013, p. 134-135) s'ouvre l'énigme de l'advenue de la subjectivité au sein du monde. Or, si la démarche de Marion consiste à rendre compte de l'ouverture phénoménale du sujet en remontant en-deçà de cette corrélation pour décrire ce qui la rend possible, ce dernier semble cependant se refuser à trouver cette origine dans une cosmologie ${ }^{13}$, ce qui lui permet d'éluder l'énigme d'une activité pure

12 Celui-ci est l'expérience d'une négativité, d'une insatisfaction qui ouvre le monde comme monde, et dont on a vu qu'il avait des conditions ontologico-métaphysiques de possibilité. Voir : (BARBARAS, 2008, p. 280- 367 ; BARBARAS, 2013, p. 115.129). Sur la relation désir-sentiment, voir: (BARBARAS, 2016, p. 256-261).

${ }^{13}$ Il faudrait cependant nuancer quelque peu cette dernière remarque. Marion tend à rejeter l'idée que l'horizon, et donc le monde, comme horizon des horizons, soit la condition de possibilité de l'apparaître des phénomènes saturés, puisqu'il en serait plutôt, en vertu de son antériorité délimitatrice, la condition d'impossibilité (MARION, 2013, p. 304-308 et 344- 348). Il est vrai que le phénomène saturé suppose malgré tout cet horizon puisqu'il le sature. Cependant, la lecture que Marion réalise de Patočka, et qu'il hérite en partie de Emilie Tardivel, le conduit à reconnaître que le monde, en tant qu'il a une structure événementiale, est un phénomène saturé. Voir (TARDIVEL, 2011, p. 269 ; MARION, 
"s'invertissant" en passivité, sans renoncer pour autant à rendre compte de la dimension événementiale des phénomènes qui nous adviennent.

Comment en effet comprendre la perception depuis la perspective ouverte par la phénoménologie de la donation? Celle-ci suppose, comme chez Barbaras (BARBARAS, 2009), la méditation de la chair. En effet, la chair fait partie des phénomènes saturés. Elle est celui d'entre eux qui sature la catégorie de relation ${ }^{14}$. Elle n'obéit donc pas au régime de la relation qui permet d'unir les objets du monde et elle échappe à la structure d'horizon. En elle, l'intuition va toujours plus loin ou donne toujours davantage que toute anticipation de sens. Ainsi, par exemple, la douleur n'est pas un objet du monde mais cela que je vis comme telle, à quoi je m’identifie immédiatement et dont le sens n'est pas clair. On est ici très proche de la description henryenne de la chair qui se donne dans l'auto-affection (HENRY, 2003, p. 105-121) et nulle part ailleurs. Elle est invisible, c'est un absolu acosmique. On ne saurait comprendre que les objets du monde puissent m'affecter, si l'on ne reconnaissait pas au préalable ce pouvoir d'auto-affection. Comme le note Marion (2001, p. 110): “[...] le propre de ma chair tient à sa souffrance, sa passivité et sa réceptivité, qui ne sont pas du monde". Pour que la conscience vise un objet, elle doit avant tout recevoir une matière sensitive qu' elle pourra informer, à laquelle elle donnera sens, mais une telle réceptivité, un tel "se laisser affecter", n'est possible que parce que la conscience s'affecte elle-même. C'est ainsi, par exemple, que la cire, comme chose du monde, peut m'apparaître. C'est parce que je suis chair, union d'une âme et d'un corps, et donc sensible que l'objet m'apparaît et non parce qu'il cause en moi des impressions.

On ne peut cependant échapper à un certain malaise en présence d'une telle description de la perception. L'accent henryen de cette thèse consonne mal avec la phénoménologie de Marion. Alors en effet que Henry reconnaît l'existence d'un dualisme phénoménologique (l'immanence invisible de

2016, p. 102). Par ailleurs, pour que le sujet puisse répondre à l'exigence de phénoménalisation de ce monde, pour que cette donation donne lieu à une monstration, il doit être incarné, être du côté du monde en tant que saturé. Or Marion ne semble pas penser véritablement l'inscription du sujet dans le monde, son être cosmologique-contrairement à Patočka - et si, d'une part, il accepte l'idée que la chair soit co-condition de la monstration et si, d'autre part, il semble souscrire à la conception patočkienne du corps comme réalisateur (MARION, 2016, p. 143), il semble plutôt hériter, par ailleurs et jusqu’à un certain point, de la conception acosmologique de la chair henryenne. Voir: (CIOCAN et VASILIU, 2016, p. 375). Sur ces premiers questionnements qui devront recevoir une élaboration ultérieure, voir (Référence effacée).

${ }^{14}$ Marion élabore sa conception de la subjectivité adonnée à partir d'un dépassement des catégories kantiennes. Voir (MARION, 2013, p. 329). 
l'auto-affection et la transcendance du monde), Marion au contraire assume un monisme phénoménologique, celui-ci fût-il susceptible de gradation (il y a des degrés de donation.) Dès lors, on voit mal comment la chair pourrait se retirer du monde pour le faire paraitre sans perdre sa connivence phénoménale avec lui. Il faut bien que la chair soit aussi du monde pour le faire paraître. Au fond, lorsque Marion évoque l'invisibilité de la chair, il faut l'entendre au sens de cela qui échappe, en tant que phénomène saturé, à la visibilité de l'objet ou de l'étant. La chair ne s'exonère pas d'une première donne du champ phénoménal mais dans la mesure où elle est l'opérateur de sa monstration pour le sujet, elle échappe au régime d'apparaître de cela qu'elle contribue à montrer ${ }^{15}$. Le sentir de la chair et la perception des objets du monde supposent tous deux une première donne phénoménale. Celle-ci est cela qui rend à la fois possible l'auto-affection d'un sujet charnel et la perception, puisque le sujet ne peut se recevoir lui-même (dans l'“auto"-affection) qu'en montrant le monde et en le recevant ${ }^{16}$. Il ne s'agit pas ici de déduire une forme de passivité à partir d'un archi-mouvement par le moyen d'un archi-événement mais de reconnaitre que le sujet - qui présuppose une première donne du mondeest co-générateur de la perception par le biais de synthèses passives (passives au regard d'un sujet constituant mais dynamiques en elles-mêmes) qui lui permettent d'organiser ce qu'il reçoit de son affection par le monde.

Si donc Marion ne semble pas rechercher l'origine de la subjectivité charnelle en tant que phénoménalisante ailleurs que dans cette première donne du monde, et non dans une ontologie cosmologique ouvrant à une métaphysique, c'est parce que ma naissance constitue par elle-même "un archi-phénomène" (MARION, 2010, p. 297) en-deçà duquel il serait vain de vouloir remonter. Marion définit la naissance comme ce phénomène ordinaire qui est pourtant aussi pur évènement (MARION, 2010, p. 293) et qui atteste de l'événementialité originaire - le pur se donner - à partir duquel le se montrer pour un sujet sera possible (MARION, 2010, p. 297). Ma naissance - qui est celle d'une vie, c'est-à-dire d'une chair - se donne sans se montrer (je n'en fais pas l'épreuve subjective ni n'en ai une connaissance objective), parce qu'elle provient d'une première manifestation vis-à-vis de laquelle elle est toujours, par définition, en retard. Elle m’échappe en conséquence du fait premier de

\footnotetext{
15 "si la naissance intervient avant le monde, ou plus exactement introduit le vivant dans un champ que la lumière du monde n'éclaire pas comme elle y éclaire les étants autrement naturels et les objets non naturels, alors la naissance reste aussi invisible que le vivant qu'elle délivre" (MARION, 2010, p. 297).

${ }^{16}$ Nous assumons ici, dans l'attente d'une élaboration ultérieure, que la première donne phénoménale est celle du monde comme totalité de ce qui peut advenir et donc comme phénomène saturé.
} 
la donation. Je phénoménalise - par moi se montre cela qui provient d'une première donne du monde - parce que moi-même je proviens de cette donne première et me reçois seulement de recevoir cela que je montre. Ma naissance atteste de l'émancipation de tout phénomène à l'égard d'une subjectivité constituante. L'impossibilité où je suis de voir l'origine en vertu de laquelle je peux advenir à moi-même tout en n'ayant aucun doute à l'égard de la possibilité permanente de cet advenir (puisqu'elle se donne à moi) donne à tout paraître, pour qui sait voir, l'allure d'une surprise ou la forme d'une "anarchie" (MARION, 2010, p. 298). Ma naissance est ce phénomène qui se donne comme événement et qui atteste de l'événement dont elle provient. Les caractères de cette manifestation (donation sans monstration, événement sans objectivité), ce retard permanent de mon advenir à moi-même sur l'origine de ce pouvoir atteste également de l'origine du devenir de la phénoménalisation qui présuppose l'événement premier de la manifestation en soi. Comme l'écrit Marion: "la naissance met en œuvre l'événementialité qui soutient et déclenche tout phénomène comme un événement qui se passe." (MARION, 2010, p. 298) C'est à travers ce phénomène événemential de la naissance que s'atteste, de manière originaire, la première manifestation du monde, mais c'est en lui aussi que se clôt la possibilité de remonter en-deçà pour penser une archéologie de la naissance.

Si ces dernières analyses sont exactes, il faut donc reconnaitre, à titre de conclusion, qu'en dépit d'un vœu commun de reconduire la phénoménologie à sa vocation première, d'en effectuer la catharsis (PATOČKA, 2002, p. 184), comme dirait Patočka, pour décrire la manifestation en soi, et de comprendre le sujet comme cela à qui advient la phénoménalité au lieu de le concevoir comme ce qui la constitue, Marion et Barbaras se séparent néanmoins, non pas tant pour ce qui concerne le caractère fondamental de l'incarnation du sujet qu'à propos de son archéologie. Dans un cas, on reste dans un cadre où la phénoménologie (de la donation) semble se suffire à elle-même, tandis que dans l'autre il faut remonter jusqu'à un plan cosmo-ontologico-métaphysique pour en rendre compte. La question du statut à donner à la naissance du sujet deviendrait alors à cet égard primordiale.

POMMIER, E. The phenomenological difference according to Barbaras and Marion (Project, method and tension line). Trans/form/ação, Marília, v. 43, n. 3, p. 111-136, Jul./Set., 2020. 


\begin{abstract}
This paper aims at giving the conditions of a confrontation between the phenomenology of the givenness of Jean-Luc Marion and the ontology of life of Renaud Barbaras. The project of the description of the appearing as such, the method, the "evential" conception of the phenomenon and the subject may constitute a plane of convergence previous to putting emphasis on a divergence between them about the question of the transcendental birth of the subject.
\end{abstract}

Keywords: Appearing. Birth. Difference. Event. Reduction.

\title{
BIBLIOGRAPHIE
}

BARBARAS, Renaud. L'être et la manifestation. In BARBARAS, Renaud . Le mouvement de l'existence, Études sur la phénoménologie de Jan Patočka. Paris: La transparence, 2007.

BARBARAS, Renaud. La perception. Paris: Hatier, 1994, Vrin, 2009.

BARBARAS, Renaud. Introduction à une phénoménologie de la vie. Paris: Vrin, 2008. BARBARAS, Renaud. Dynamique de la manifestation. Paris: Vrin, 2013.

BARBARAS, Renaud. Métaphysique du sentiment. Paris: Cerf, 2016.

CIOCAN, Cristian. et VASILIU, Anca. (eds). Lectures de Jean-Luc Marion. Paris: Cerf Patrimoines, 2016.

FRANCK, Didier. Dramatique des phénomènes. Paris: PUF, 2001.

HENRY, Michel. Phénoménologie de la vie I. De la phénoménologie. Paris: PUF, 2003.

HUSSERL, Edmund. Idées directrices... I, \$ 24, Hua. III, tr.fr., P. Ricoeur, Paris, 1950.

JACQUET, Frédéric. Métaphysique de la naissance. Louvain-la-Neuve: Peeters, 2018.

JANKELEVITCH, Vladimir. Philosophie première. Paris: Quadrige PUF, 1986

MARION, Jean-Luc. De Surcroît. Paris: PUF, 2001.

MARION, Jean-Luc. Certitudes négatives. Paris: Figures Grasset, 2010.

MARION, Jean-Luc. Etant donné. Paris: P.U.F, 2013.

MARION, Jean-Luc. Reprise du Donné. Paris: PUF, 2016.

MONTAIGNE, Michel. Essais III. Paris: PUF, 1965.

PATOČKA, Jan. Qu'est-ce que la phénoménologie? Grenoble: Millon, 2002.

ROMANO, Claude. Remarques sur la méthode phénoménologique dans Étant donné.

Annales de Philosophie, vol. 21, Université Saint-Joseph, Beyrouth, 2000.

TARDIVEL, Emilie. La liberté au principe. Paris: Vrin, 2011. 
POMMIER, E

TARDIVEL, Emilie. Monde et donation. Une révision du quatrième principe de la phénoménologie. Revue de métaphysique et de morale. Paris: Puf, 2015.

Recebido: 14/08/2018

Aceito: 23/02/2020 